\title{
Relationship Between Heroin Addiction and Depression: Gender Difference and Treatment
}

\author{
Ming Cheng ${ }^{1, *}, \dagger$, Chuanxi $\mathrm{Li}^{2, \dagger}$, Jiayu $\mathrm{Xie}^{3, \dagger}, \mathrm{Zi} \mathrm{Ye}^{4, \dagger}$ \\ ${ }^{1}$ University of Ottawa, Ottawa, Ontario, Canada \\ ${ }^{2}$ Hangzhou N0.14 Middle School, Hangzhou, Zhejiang, China \\ ${ }^{3}$ Jiangxi University of Technology High School, Nanchang, Jiangxi, China \\ ${ }^{4}$ Beijing Huijia Private School, Beijing, Beijing, China \\ *Corresponding author.Email: mchen242@uottawa.ca \\ These authors contributed equally.
}

\begin{abstract}
Heroin addiction and depression have recently become more serious, seriously affecting people' $\mathrm{s}$ lives. The relationship between them has been well discovered, but the role gender plays in this relationship remains unclear. Therefore, the purpose of this paper is to discuss the relationships between heroin addiction, depression and their treatments, and the role of gender differences in these relationships. Firstly, heroin addiction and depression are separately introduced, including their impacts, gender difference and treatments for heroin addiction. Reviewing previous literature shows a strong correlation between heroin addiction and depression, and they influence and contribute to each other. This relationship leads to our focus on patients with both heroin addiction and depression. We find that gender plays a role in influencing the level of both diseases and the outcomes of treatments, showing that women have higher depression scores before treatments and their depression scores decrease faster after treatments. In conclusion, heroin addiction and depression are both influenced by gender, and women and men react differently to treatments, so future research should consider this factor when choosing treatments. Moreover, treatments should be studied and categorized to fit both genders' specific needs.
\end{abstract}

Keywords: Heroin Addiction, Depression, Gender Difference, Treatment.

\section{INTRODUCTION}

Drug addiction is a chronic illness that causing people to continuously crave drugs despite the harmful effect of drug use [1]. Specifically, the use of drugs can create a feeling of pleasure, or "high", and lead to physical and psychological addiction by activating the brain reward system and changing the way of brain functioning $[1,2]$.

One of the common drugs is heroin, which is a highly addictive opiate substance. Similar to all other drugs, heroin use has many detrimental influences, and it already become very popular. Many studies have found heroin addiction to be an increasingly serious concern worldwide $[3,4]$. It is reported that the rate of heroin use and heroin addiction increased significantly in recent years. As a result, there is also an increase in the number of deaths related to heroin overdose [4]. Moreover, heroin addiction can bring many devastating consequences to the addicts, including health impairment, financial difficulties, social problems, etc. In the meantime, it has a strong adverse effect on the psychological state of heroin users, so it is associated with many mental disorders [5].

Specifically, researchers have noticed depression as an important psychological issue among heroin users [6, 7]. It is a common mental illness characterized by losing interest in daily activities, feelings of sadness, and so on [1]. Several studies concluded a substantially higher prevalence rate of major depression among heroin users than the general population [6,7]. This might indicate a potential relationship between heroin addiction and depression. Additionally, to help heroin addicts recover from addiction and related depressive symptoms, many treatments are adopted. These treatments are proved to influence reducing addiction and depressive levels of heroin users [7]. 
Furthermore, it is remarkable that gender plays an essential role in heroin addiction, depression, and treatments [3, 8]. However, few studies have clearly demonstrated the relationship between these variables and how gender moderates this relationship. Therefore, we developed our aim to discuss the relationships between heroin addiction, depression and their treatments, and the role of gender differences in these relationships. We believe that these are important to understand since they can help people better prevent the detrimental effects of heroin addiction and depression on both individual users and society.

In this review, to clarify the research questions, we will first introduce heroin addiction and depression by discussing their impacts and the role of gender differences in them separately. Then, the treatments for heroin use will be discussed. At last, we will talk about the relationships between these variables and how gender may affect these relationships.

\section{HEROIN ADDICTION}

\subsection{Impact of Heroin Addiction}

About heroin addicts, heroin affects them physically and psychologically at the same time. Aksu et al. [9] found that somatization was more prevalent in heroin addicts, whose SCL-90 subscales somatization values are even higher than alcohol addicts. They are more likely to feel serious discomforts such as headaches, dizziness, insomnia or nausea, but cannot be detected as having significant pathological changes from medical examination. At the psychological level, heroin addicts will also have higher levels of negative emotions such as anger, hostility towards others and interpersonal sensitivity [9]. Individuals who use heroin on a longterm basis are more likely to exhibit multiple physical and mental health disorders, including depression, anxiety, hepatitis, and tuberculosis [10].

At the same time, the increase in heroin addicts will also have some social effect. Firstly, regarding crime rates, heroin addicts may choose to commit crimes to get more money to buy drugs [11]. Moreover, this would have a great social cost. Treatment for heroin use disorders, chronic infectious diseases caused by heroin and their treatment, overdose deaths from heroin, neonatal cut-off syndrome, and loss of productivity are all costly to address. In the United States alone, the social cost of heroin addiction is $\$ 51.2$ billion (in 2015 dollars), or an average of $\$ 50,799$ per heroin addict [12].

In conclusion, heroin addiction can cause psychological and physical illness and many social problems and costs to society.

\subsection{Formation of Gender Difference and Its Impacts on Heroin Addiction}

The addiction of heroin for both males and females has devastating consequences. However, there are significant differences in the development of heroin addiction between men and women due to biological and social effects. Through the data statistics of clinical patients, it can be learned that there is diversity in the subtle biological foundation of male and female, initial marriage condition for male and female patients, the time of first drug attempt, the time of the first treatment attempt and whether the rehabilitation course can be completed well [13]. Therefore, an in-depth study on the relationship between gender factors and heroin addiction is of practical significance for preventing subsequent drug use and the treatment of addicted patients.

\subsubsection{Biological Aspects}

There is growing evidence that there are biologically-based sex differences at every stage of drug addiction in both humans and laboratory animals [1416]. Sex differences in the developmental origin and expression of the nervous system may lead to sex differences in addictive behaviours. Although there is evidence that many qualitative aspects of the addiction stage do not differ significantly between men and women [16]. However, according to multiple studies, the biology of pain and opioid addiction is different in women and men [14, 15]. Focusing on both similarities and differences between men and women in the biological aspects in addiction progress will improve the feasibility of prevention and treatment of addiction disorders.

In substance abuse, the differences in the number of users and the increase in substance abuse were marked by gender. Based on the prevalence of the total sample from 2007 to $2014(\mathrm{~N}=447,188)$, while non-medical use of prescription opioids declined steadily, heroin use became dramatically famous for both men and women, and female heroin use increased at a faster rate than males [15]. It can be said that there was a linear increase in heroin use and a marked difference in rates of change between men and women in the use of heroin [8]. Currently, researchers have highlighted the associations of four well-known key neurological factors with addiction: dopamine, mu-opioid receptor (MOR), kappa opioid receptor (KOR), and brain-derived neurotrophic factor (BDNF) and how they interact with gender [16]. Specific chemical secretions in the body activate the brain and nervous system in gender-specific ways: sex discrepancy plays a vital role in the regulation of the dopamine system; specific genotypes of MOR are associated with the risk of a heroin use disorder, with gender differences that have not yet been identified; 
KORs creates a difference in how men and women experience addiction, and BDNF receptor may be responsible for the plasticity of emotional and motivational circuits, affecting addictive behaviors [16]. In addition, gonadal hormones also impact the relationship between neural function and sex difference, which is sensitive to the stage of addiction $[16,17]$. Furthermore, when female subjects were placed in the experimental group in rodents, the results showed gender differences in the nervous system's modulation of pain response and opioid-related pain reduction [17]. On the other hand, when subjects with opioid dependence were exposed to the laboratory stress paradigm, women reported greater subjective stress than men [14]. Even though women were less responsive to cortisol in stressful tasks, the increased subjective response of both the inactive cortisol and women may be markers of HPA axis disorder, increasing women's vulnerability to relapse [14]. In experiments that examined more than just basic physiological differences, it was found that women were twice as likely as men to develop mood disorders or anxiety disorders and that people with lifelong mental disorders were approximately three times as likely to rely on other substances, regardless of gender [18]. All the above concepts highlight the importance of gender-sensitive prevention and treatment approaches.

\subsubsection{Sociological Aspects}

For heroin patients, they face many of the same environmental issues, such as family relations, financial resources, and social condemnation [13]. However, gender differences make men and women affected differently by these environmental issues. The financial sources of heroin use are different, which also confirmed gender differences. Compared to men, family support or partners' savings are often a vital source for female heroin purchases [19]. And the original family also affects heroin use differently in men and women [20]. A huge proportion of female patients come from dysfunctional families of origin. In the family formed by single patients, the probability of male patients living with their children is lower than that of female patients [20].

In conclusion, female addicts were more likely to have experienced childhood trauma, such as sexual abuse and violence, and were more likely to be married and have children. Meanwhile, using heroin with their husbands or partners is reported by women. Those compare the different previous situations which lead males and females to use heroin.

\subsection{Treatments for Heroin Addiction}

Two main treatments are frequently used for heroin addiction: pharmacological treatment and rehabilitation
$[6,21,22]$, and they are still widely used today. The pharmacological treatment provides medicine to treat opioid dependence, including detoxification and methadone maintenance treatment (MMT). Rehabilitation does not contain medicine, but it treats opioid users by arranging psychological counselling and psychotherapy. In this paper, we mostly focus on MMT and psychological counselling.

\subsubsection{Methadone Maintenance Treatment}

Methadone maintenance treatment (MMT) has been used to treat opioid dependence since the 1960s. It is one type of opioid maintenance treatment (OMT), the most commonly used treatment for heroin addiction [23]. After decades of development and application, MMT is still being proved valid and used in many current studies to treat heroin addiction $[23,24]$. Today, heroin addicts in MMT are often engaged in the research that discovers the correlated factor of heroin addiction, like loneliness $[24,25]$ and pain [24].

\subsubsection{Psychological counselling}

Counselling is a commonly used rehabilitation method for opioid dependence users. This type of treatment is usually associated with pharmacological treatments and interact with each other to influence the outcome of treatments. Peterson et al. [21] concluded that opioid use hospital inpatients in the US received both detoxification and rehabilitation as treatments, and the discharge rate of inpatients increased from 1993 to 2016. Researchers also found that there were interactions between these two types of treatments. Research conducted in 2018 reported that patients receiving both educational and behavioural counselling (EBC) and MMT had better attendance and knowledge of heroin addiction than those who only received MMT [26]. Li et al. [24] also found the relationship between counselling and loneliness when they were researching the pain level in MMT.

\section{DEPRESSION}

\subsection{Impact of Depression}

At the present time, more people no longer have to worry about food and clothing, and psychological problems are becoming more of a concern, the most widely known of which is depression. People are more concerned about their mental health, but the number of people suffering from depression is increasing due to the increasing social pressure, with teenagers accounting for a large proportion of depressed people.

Depression can have several effects on a person's health. It can be more harmful to specific groups of people. For example, when women with a major depressive disorder are pregnant, although they have the 
same symptoms as non-depressed pregnant women, their suicidal tendencies, guilt, and early insomnia are more severe [27]. Even after treatment, the vast majority of older people with major depression are more likely to have residual symptoms of depression, which can also cause them [28].

\subsection{Formation of Gender Difference and Its Impacts on Depression}

The former data convinced that heroin addicts have a greater possibility of fighting mental disease and symptoms simultaneously. In this regard, gender is a major influence factor, reporting that there are distinguishable differences between males and females under the condition of the same kind of mental disease. From last century, scholars have underscored the gender difference in depression based on the clinical and community samples, showing that nearly twice as a number of women experience depression as men [29]. As more and more research emerges and society changes, it is also critical to take a developmental perspective. According to Rungreangkulkij [30], the scholar states that depression is one of the most common disorders affecting women in each nation, highlighting that gender is a vital determinant of mental health. Meanwhile, the paper has shown that the effect of depression on all-cause mortality is stronger in men even though the influence of the age factor is still unclear [31]. Therefore, it can be said that there are a variety of different factors that contribute to gender differences in the relationship between depression and gender. Differences in age, biology, socio-cultural background and race are all variables to gender differences in the impact and proportion of depression, especially biological foundation and social environment contributing to the gender difference in depression.

\subsubsection{Biological Aspects}

Women are twice as likely as men to be diagnosed with depression, and there are physical sex differences in symptoms, severity, and antidepressant response [32]. Regardless of the interplay of biological, psychological, and socio-cultural influences, there are fundamental physiological differences in the brain and nervous system between men and women that play a role in producing and influencing emotions, especially negative ones. Based on the study, higher CRP levels were associated with greater depressive symptom severity in women $(\mathrm{P}=0.02)$. In contrast, higher $\mathrm{C}$-reactive protein levels were associated with observed increases in mood, cognitive symptoms, the activity of interest, and the severity of suicide but were not significant in men $(\mathrm{P}=$ 0.68) [33]. There was some consistency in how often depressive symptoms occurred and to what extent they were diagnosed between men and women at each age. For example, adolescence is a critical period for the development of depression. The data of a national survey on adolescents aged 12-17 in the United States $(\mathrm{N}=101685)$ for 6 consecutive years (2009-2014) were used to describe the gender difference in the incidence of depression by age. The incidence ratio of specific age was calculated [34]. The cumulative incidence of depression between the ages of 12 and 17 was $13.6 \%$ in males, and $36.1 \%$ in females and incidence was significantly higher at ages 13 to 17 than at ages 12 (P $<0.05)$, ), which means biological mechanism contributes to this stark sex difference. Neuroendocrine factors, particularly the regulation of mood by sex hormones and the development of brain organs during adolescence as the peak of gender differentiation, vary the likelihood of depression in both men and women [35]. Also, it is obvious that elderly women had significantly more depressive symptoms than men [32].

Compared to heroin addiction, the gender difference in depression reveals a health disparity, especially in adolescence. Yet, no matter for female or male, the magnitude of the difference demonstrates that depression should not be overlooked.

\subsubsection{Sociological Aspects}

From the perspective of sociology, the gap between rich and poor and gender inequality leads to gender differences, which have a more significant impact on depression, such as the artificial elimination of discrimination against women in society, the stereotype of men and women, and the lower social status of women compared to the majority of men [13]. However, the gender difference in depression is smaller in countries and regions where gender equality is valued and practiced to some extent [36]. As for the pooled sample by Shervin Assari [13] showed that gender interacted with the effects of income on major depressive episodes, suggesting that the association between household income and major depressive episodes is larger for women than men.

In race by gender-specific models that controlled for other SES indicators, as for African American men and white women, high income could be the risk factor for the former and the protective factor for the latter [13]. Evaluating the magnitude of gender differences in depression, women have higher major depression and depression symptoms than men. Such a stereotype can be harmful to both women and men.

\section{RELATIONSHIPS}

\subsection{Relationship between Heroin Addiction and Depression}

The relationship can be separated into two parts: How heroin addiction can affect depression and how depression can influence heroin addiction. 
Firstly, when a person is addicted to heroin, they will be more likely to suffer from depression than those who do not take heroin. From a neurobiological perspective, Kosten et al. [37] found that two crucial neurotransmitter systems that undergo physiological changes due to chronic drug use are serotonin and dopamine, which contribute to depression and substance abuse in vulnerable individuals. Subsequently, the serotonergic system becomes disorganized and reacts to the dopamine effect of the drug, making heroin users more susceptible to depression [37]. Next, Seifert et al. [38] found that people who were chronically dependent on heroin had a reduction in the volume of the nucleus accumbens in their brains, which led to an increased incidence of depression in them. Meanwhile, people with depression are also more likely to use drugs and become addicted. Their depression levels are usually several times higher than those of the general population [6]. They are more likely to be caught up in negative emotions and thus more likely to use drugs to relieve their feelings.

In conclusion, there is a strong correlation between heroin addiction and depression, and they influence and contribute to each other. Individuals relieving depression state by taking heroin, and then the heroin will make their depression worse.

\subsection{Overview for Gender Difference}

Based on former relevant papers, it is believed that male and female difference is significant in causal factors of heroin addiction and depression and influential to the initiation of heroin use and the sequence of the treatments.

According to the 2010 U.S. Census, $50.8 \%$ of women have an addiction disorder [16]. From various studies in this field, there is a significant disparity between the sexes in terms of the purpose of use, the biology of addiction, the triggers of addiction, the experience of withdrawal, and the likelihood of relapse [16-18]. On the other hand, the gender gap in depression is obvious. The fact that women are much more inclined to be diagnosed as depressed than men reflects gender differences in neural and physiological basis, social environment, age, and many more factors that have not been fully explored [32-34]. Even though there are some unavoidable variations, the total results showed that the main factors basically impact the gender gap for heroin addiction and depression.

The causes of drug addiction are still complicated. So does the cause of depression. Individual differences, especially differences between gender, have a significant impact on the formation of addiction and the behaviour patterns displayed. Rather than finding one single determinant, scientists have identified several psychological and social-cultural preconditions as precursors of addiction and depression, demonstrating the relationship of gender difference and the other two disorders.

\subsubsection{Heroin Addiction and Depression: Gender Difference}

According to available research, many heroin addicts suffer from co-existing mental disorders, such as depression, antisocial personality disorder, posttraumatic stress disorder and anxiety disorder [13]. In addition, addicts are more likely to see symptoms of anxiety and depression as well as emotional fatigue and apathy due to long-term use or withdrawal from heroin [19].

Unfortunately, for women, there was a higher occurrence of depression and anxiety [34]. After seeking a database, women have a greater possibility of utilising drugs to dissolve psychiatric problems than men [17]. Women surveyed in a methadone treatment program reported that they were more likely to use drugs to alleviate psychological distress than male patients [41]. To sum up, the prevalence of psycho problems among women highly seduces them to apply heroin as the incorrect solution to solve depression.

The gender difference among patients with both depression and opioids dependence disorder is researched, and gender is found to be correlated to many influencing factors in the relationship between heroin addiction and depression. Butelman et al. [42] had found that gender played a role in influencing depression comorbidity among opioid dependence users. They had found that women had a higher rate of conducting depression comorbidity than men [42]. Another research also found that depressive disorder in women with opioid use disorder had a higher risk of cooccurring sedative use disorder [43]. However, male heroin users with depression were inconsistently related to overdose risk in a case study [44] and their opioid craving behaviors were indirectly related to childhood trauma [45]. Similar results were found by Marchand et al. [46] while examining suicidal attempts between women and men heroin users with depression, showing that the suicidal attempts for women were affected by a history of depression while that for men were affected by childhood trauma. Although the study of DjurendicBrenesel [20] showed that women heroin users were more likely to have childhood trauma, men's suicidal attempts were still affected by it. These results show that in opioid users, women are more likely to have cooccurring mental disorders while men are more likely to have drug-related behaviors. 


\subsection{Gender and Treatment in Heroin Addiction and Depression}

Before the beginning of treatments, heroin users were commonly found to have higher depression levels than people who do not take drugs. In this case, treatments seem to play an essential role in decreasing depression levels. Many studies have found out that the depression level of heroin addicts decreased after treatments [7, 47]. Wang et al. [48] also referred that depression in the female heroin users decreased faster during MMT than in the male heroin users.

A suicidal attempt is also a critical predictor in examining depression levels among heroin users in treatment. Darke et al. [49] found that young females have the highest drug use level and suicide attempts among all participants. Also, they indicated that participants in drug-free residential rehabilitation had a higher rate of suicide attempts [50]. Another research held by Darke et al. [50] also found the relationship between suicidal rate and treatments, showing that the suicidal rate of men did not change after treatments, but that of women significantly decreased [50].

However, some researchers hold different views. The research held by Darke et al. [50] found no significant difference among treatments on their impact of depression. Meanwhile, according to Teesson et al. [6], there was a considerable difference between the treatment group and non-treatment group in the change of depression. The depression level of the treatment group was significantly higher than that of the nontreatment group. Especially, women in the treatment group had the highest depression level. These results show an adverse opinion on how treatments influence depression, indicating that there are still arguments in this aspect.

Due to different outcomes of treatments between females and males, choosing treatment becomes important, and it is affected by multiple factors. For example, Friesen and Kurdyak [51] suggested that people with a mental disorder had a lower rate of dropping out of the treatment program but were more likely to have the treatment stopped by the treatment facility early. Gender also plays a role in choosing treatments among heroin addicts. According to Zhou et al. [11], males are more likely to be in detention facilities and MMT clinics. The researchers stated that this happened because men were more likely to commit crimes and get into prison when they had run out of money to afford drugs, so they would be in detention facilities to do drug detoxification [11]. While for women, heroin addicts had more chores to do, and they did not have the time to make MMT a daily routine since this program asks people to go to the clinics regularly [11]. These results suggest that when choosing treatment for heroin dependence users, it is important to consider the co-occurring mental disorder and gender as factors that will influence the treatment outcome. However, the research on this aspect is still limited and need future discovery.

\section{FUTURE DIRECTION}

According to the previous studies, there are still some general limitations in this field of research. Firstly, the majority of the studies did not well control their variables. For example, some studies compared the result from different treatments of heroin dependence, but they did not control the type of treatment by themselves. Instead, they recruit participants that received treatments from various agencies $[6,7]$. Thus, this progress will end up with many confounding variables that may further affect the validity of the results. Secondly, some studies had assessed the level of depression or heroin addiction by asking participants to do a self-reporting questionnaire $[4,7]$. In this case, there may be social desirability bias, which may influence the assessment's accuracy. Moreover, an evident difference is found in how males and females react to the treatment of depression and heroin addiction, while few treatments that are widely used now really considered the influence of gender differences and therefore, the effectiveness of treatments might be limited [49].

To minimize the adverse effects of these problems, researchers may need some improvements. To state it more clearly, confounding variables should be controlled more strictly with standardized procedures, and a professional diagnosis is required for a more accurate assessment. Additionally, further research needs to pay more attention to the development of gender-sensitive treatments. Researchers should take gender differences into account when developing new treatments, so it may help enhance the effectiveness of treatment in both males and females.

\section{CONCLUSION}

Heroin addiction and depression both impact people's lives in many ways. Both heroin addiction and depression would impact an individual's physical and mental health, while heroin addiction would increase crime rates and financial costs. Gender plays a role in influencing both heroin addiction symptoms and depression levels. Generally, women are more likely to have higher depression levels, and men are more likely to express aggressive symptoms, and biological and sociological factors cause this gender difference. Relationship among heroin addiction, depression, gender and treatment has been discussed by focusing on individuals with heroin addiction and depression who receive treatments, and the result shows that people with the mental disorder would be stopped from keep receiving treatment by facilities early. Women and men 
show up in different types of treatments. This result shows that heroin addiction and depression are frequently co-occurring, and gender difference influences both the severity of these diseases and the outcome of treatments. Therefore, future researches should focus on the gender difference in heroin addiction treatments and further improvements are needed.

\section{REFERENCES}

[1] American Psychiatric Association. (2013). Diagnostic and statistical manual of mental disorders (5th ed.). Arlington: American Psychiatric Association.

[2] McLellan, A. T., Lewis, D. C., O'Brien, C. P., \& Kleber, H. D. (2000). Drug dependence, a chronic medical illness. JAMA, 284(13), 1689-1695. https://doi.org/10.1001/jama.284.13.1689

[3] Degenhardt, L., Bucello, C., Mathers, B., Briegleb, C., Ali, H., Hickman, M., \& McLaren, J. (2010). Mortality among regular or dependent users of heroin and other opioids: A systematic review and meta-analysis of cohort studies. Addiction, 106(1), 32-51. https://doi.org/10.1111/j.13600443.2010.03140.x

[4] Jones, C. M., Logan, J., Gladden, R. M., \& Bohm, M. K. (2015). Vital Signs: Demographic and Substance Use Trends Among Heroin Users United States, 2002-2013. MMWR. Morbidity and mortality weekly report, 64(26), 719-725.

[5] Swendsen, J., Conway, K. P., Degenhardt, L., Glantz, M., Jin, R., Merikangas, K. R., Sampson, N., \& Kessler, R. C. (2010). Mental disorders as risk factors for substance use, abuse And DEPENDENCE: Results from the 10-YEAR follow-up of the National Comorbidity Survey. Addiction, 105(6), 1117-1128. https://doi.org/10.1111/j.1360-0443.2010.02902.x

[6] Teesson, M., Havard, A., Fairbairn, S., Ross, J., Lynskey, M., \& Darke, S. (2005). Depression among entrants to treatment for heroin dependence in the australian Treatment Outcome Study (atos): Prevalence, correlates and TREATMENT SEEKING. Drug and Alcohol Dependence, 78(3), 309-315. https://doi.org/10.1016/j.drugalcdep.2004.12.001

[7] Darke, S., Mills, K., Teesson, M., Ross, J., Williamson, A., \& Havard, A. (2007). Patterns of major depression and drug-related problems amongst heroin users across 36 months. Psychiatry Research, 166(1), 7-14. https://doi.org/10.1016/j.psychres.2007.12.007
[8] Marsh, J. C., Park, K., Lin, Y.-A., \& Bersamira, C. (2018). Gender differences in trends for heroin use AND nonmedical prescription opioid use, 2007 2014. Journal of Substance Abuse Treatment, 87, 79-85. https://doi.org/10.1016/j.jsat.2018.01.001

[9] Aksu, M. H. (2020). Relationship between somatization and psychiatric symptoms, especially anxiety, depression, alexithymia, and severity of addiction in male patients with alcohol and heroin addiction. Dusunen Adam: The Journal of Psychiatry and Neurological Sciences. https://doi.org/10.14744/dajpns.2020.00071

[10] Grella, C. E., \& Lovinger, K. (2012). Gender differences in physical and mental health outcomes among an aging cohort of individuals with a history of heroin dependence. Addictive Behaviors, $37(3)$, 306-312. https://doi.org/10.1016/j.addbeh.2011.11.028

[11] Zhou, X., Yi, Z., Yang, X., Wang, Z., Lyu, X., \& Li, J. (2017). Gender Differences and Correlated Factors of Heroin Use Among Heroin Users. Substance Use \& Misuse, 52(1), 25-32. https://doi.org/10.1080/10826084.2016.1212891

[12] Jiang, R., Lee, I., Lee, T. A., \& Pickard, A. S. (2017). The societal cost of heroin use disorder in the United States. PLOS ONE, 12(5). https://doi.org/10.1371/journal.pone.0177323

[13] Assari, S. (2017). Social determinants of depression: The intersections of race, gender, and socioeconomic status. Brain Sciences, 7(12), 156. https://doi.org/10.3390/brainsci7120156

[14] Gilmore, A. K., Guille, C., Baker, N. L., Brady, K. T., Hahn, C. K., Davis, C. M., McCauley, J. L., \& Back, S. E. (2019). Gender differences in subjective stress and neuroendocrine response to a stress task among individuals with opioid dependence: A pilot study. Addictive Behaviors, 92, 148-154. https://doi.org/10.1016/j.addbeh.2018.12.022

[15] Marsh, J. C., Park, K., Lin, Y.-A., \& Bersamira, C. (2018). Gender differences in trends for heroin use AND nonmedical prescription opioid use, 2007 2014. Journal of Substance Abuse Treatment, 87, 79-85. https://doi.org/10.1016/j.jsat.2018.01.001

[16] Becker, J. B., \& Chartoff, E. (2018). Sex differences in neural mechanisms mediating reward and addiction. Neuropsychopharmacology, 44(1), 166-183. https://doi.org/10.1038/s41386-0180125-6

[17] Becker, J. B., \& Mazure, C. M. (2019). The federal plan for health science and technology's response 
to the opioid crisis: Understanding sex and gender differences as part of the solution is overlooked. Biology of Sex Differences, 10(1). https://doi.org/10.1186/s13293-018-0215-5

[18] Grella, C. E., Karno, M. P., Warda, U. S., Niv, N., \&amp; Moore, A. A. (2009). Gender and comorbidity among individuals with opioid use disorders in THE Nesarc study. Addictive Behaviors, 34(6-7), 498-504. https://doi.org/10.1016/j.addbeh.2009.01.002

[19] Zhao, Q., Li, H., Hu, B., Li, Y., Gillebert, C. R., Mantini, D., \& Liu, Q. (2018). Neural correlates of drug-related attentional bias in heroin dependence. Frontiers in Human Neuroscience, 11. https://doi.org/10.3389/fnhum.2017.00646

[20] Djurendic-Brenesel, M., \& Pilija, V. (2016). Opiate receptors and gender and relevance to heroin addiction. Neuropathology of Drug Addictions and Substance Misuse, 922-932. https://doi.org/10.1016/b978-0-12-8002131.00086-9

[21] Peterson, C., Xu, L., Florence, C., \& Mack, K. A. (2019). Opioid-related US hospital discharges by type, 1993-2016. Journal of Substance Abuse Treatment, 103, 9-13. https://doi.org/10.1016/j.jsat.2019.05.003

[22] Zhang, H.-H., Tan, L.-X., Hao, W., \& Deng, Q.-J. (2017). Evaluation of a community-based integrated heroin addiction treatment model in Chinese patients. Oncotarget, 8(33), 54046-54053. https://doi.org/10.18632/oncotarget.18681

[23] Kriegler, J., Wegener, S., Richter, F., Scherbaum, N., Brand, M., \& Wegmann, E. (2019). Decision making of individuals with heroin addiction receiving opioid maintenance treatment compared to early abstinent users. Drug and Alcohol Dependence, 205, 107593-107593. https://doi.org/10.1016/j.drugalcdep.2019.107593

[24] Li, F., Xu, Y.-M., Zhu, J.-H., Lu, J., \& Zhong, B.-L. (2017). Pain of methadone-maintained heroin addicts: lonelier individuals feel more intense pain. Oncotarget, 8(45), 79948-79952. https://doi.org/10.18632/oncotarget.20387

[25] Yang, Y.-J., Xu, Y.-M., Chen, W.-C., Zhu, J.-H., Lu, J., \& Zhong, B.-L. (2017). Loneliness and its impact on quality of life in Chinese heroindependent patients receiving methadone maintenance treatment. Oncotarget, 8(45), 7980379808. https://doi.org/10.18632/oncotarget.19565

[26] Liu, P., Song, R., Zhang, Y., Liu, C., Cai, B., Liu, X., Li, J., Chen, X., Ke, J., Lou, J., Chen, W., Zhu,
B., Zou, L., Yang, Y., Zhu, Y., Gong, Y., Zhong, R., \& Miao, X. (2018). Educational and Behavioral Counseling in a Methadone Maintenance Treatment Program in China: A Randomized Controlled Trial. Frontiers in Psychiatry, 9, 113113. https://doi.org/10.3389/fpsyt.2018.00113

[27] Manber, R., Blasey, C., \& Allen, J. J. B. (2008). Depression symptoms during pregnancy. Archives of women's mental health, 11(1), 43-48.

[28] Gastó, C., Navarro, V., Catalán, R., Portella, M. J., \& Marcos, T. (2003). Residual symptoms in elderly major depression remitters: Residual symptoms in depression. Acta Psychiatrica Scandinavica, 108(1), 15-19. https://doi.org/10.1034/j.1600-0447.2003.00068.x

[29] Tiwari, A., \&amp; Gonzalez, A. (2018). Biological alterations affecting risk of adult psychopathology following childhood trauma: A review of sex differences. Clinical Psychology Review, 66, 6979. https://doi.org/10.1016/j.cpr.2018.01.006

[30] Rungreangkulkij, S., Kotnara, I., Rujiraprasert, N., \& Khuandee, N. (2019). Gender Inequality Identified as an Underlying Cause of Depression in Thai Women. Journal of International Women's Studies, 20(7), 394-.

[31] Patten, S. B. (2012). Recurrence risk in major depression. Depression and Anxiety, 30(1), 1-4. https://doi.org/10.1002/da.22030

[32] Girgus, J. S., Yang, K., \& Ferri, C. V. (2017). The Gender Difference in Depression: Are Elderly Women at Greater Risk for Depression Than Elderly Men?. Geriatrics (Basel, Switzerland), 2(4), 35.https://doi.org/10.3390/geriatrics2040035

[33] Köhler-Forsberg, O., Buttenschøn, H. N., Tansey, K. E., Maier, W., Hauser, J., Dernovsek, M. Z., Henigsberg, N., Souery, D., Farmer, A., Rietschel, M., McGuffin, P., Aitchison, K. J., Uher, R., \&amp; Mors, O. (2017). Association between C-reactive protein (crp) with depression symptom severity and SPECIFIC depressive symptoms in major depression. Brain, Behavior, and Immunity, 62, 344-350. https://doi.org/10.1016/j.bbi.2017.02.020

[34] Breslau, J., Gilman, S. E., Stein, B. D., Ruder, T., Gmelin, T., \&amp; Miller, E. (2017). Sex differences in recent first-onset depression in an EPIDEMIOLOGICAL sample of adolescents. Translational Psychiatry, 7(5). https://doi.org/10.1038/tp.2017.105

[35] Naninck, E. F. G., Lucassen, P. J., \& Bakker, J. (2011). Sex Differences in Adolescent Depression: Do Sex Hormones Determine 
Vulnerability? Journal

of

Neuroendocrinology, 23(5),

383-392.

https://doi.org/10.1111/j.1365-2826.2011.02125.x

[36] Salk, R. H., Hyde, J. S., \& Abramson, L. Y. (2017). Gender differences in depression in representative national samples: Meta-analyses of diagnoses and symptoms. Psychological Bulletin, 143(8), 783822. https://doi.org/10.1037/bul0000102

[37] Kosten, T. R., Markou, A., \& Koob, G. F. (1998). Depression and stimulant dependence: Neurobiology and pharmacotherapy. The Journal of Nervous and Mental Disease, 186(12), 737-745.

[38] Seifert, C. L., Magon, S., Sprenger, T., Lang, U. E., Huber, C. G., Denier, N., Vogel, M., Schmidt, A., Radue, E.-W., Borgwardt, S., \& Walter, M. (2015). Reduced volume of the nucleus accumbens in heroin addiction. European Archives of Psychiatry and Clinical Neuroscience, 265(8), 637-645. https://doi.org/10.1007/s00406-014-0564-y

[39] Chatham, L. R., Hiller, M. L., Rowan-Szal, G. A., Joe, G. W., \&amp; Simpson, D. D. (1999). Gender differences at admission and follow-up in a sample of methadone MAINTENANCE Clients. Substance Use \&amp; Misuse, 34(8), 1137-1165. https://doi.org/10.3109/10826089909039401

[40] Butelman, E. R., Bacciardi, S., Maremmani, A. G. I., Darst-Campbell, M., Correa da Rosa, J., \& Kreek, M. J. (2017). Can a rapid measure of selfexposure to drugs of abuse provide dimensional information on depression comorbidity? The American Journal on Addictions, 26(6), 632-639. https://doi.org/10.1111/ajad.1257

[41] Ellis, J. D., Pittman, B. P., \& McKee, S. A. (2020). Co-occurring opioid and sedative use disorder: Gender differences in use patterns and psychiatric co-morbidities in the United States. Journal of Substance Abuse Treatment, 114, 108012-108012. https://doi.org/10.1016/j.jsat.2020.108012

[42] Tas, B., Jolley, C. J., Kalk, N. J., Waal, R., Bell, J., \& Strang, J. (2020). Heroin-induced respiratory depression and the influence of dose variation: within-subject between-session changes following dose reduction. Addiction (Abingdon, England), 115(10),

1954-1959. https://doi.org/10.1111/add.15014

[43] Ghorbani, F., Khosravani, V., Mohammadzadeh, A., \& Shadnia, S. (2019). The role of emotion dysregulation in the relation of childhood trauma to heroin craving in individuals with heroin dependence. Drug and Alcohol Dependence, 195, $132-139$.

https://doi.org/10.1016/j.drugalcdep.2018.12.008
[44] Marchand, K., Palis, H., Fikowski, J., Harrison, S., Spittal, P., Schechter, M. T., \& Oviedo-Joekes, E. (2017). The Role of Gender in Suicidal Ideation among Long-term Opioid Users. Canadian Journal of Psychiatry, 62(7), 465-472. https://doi.org/10.1177/0706743717711173

[45] Chen, W.-T., Wang, S.-C., Wang, I.-A., Tsay, J.-H., \& Chen, C.-Y. (2020). Suicide attempts and death among heroin-involved women seeking methadone treatment in Taiwan. Drug and Alcohol Dependence, 217, 108277-. https://doi.org/10.1016/j.drugalcdep.2020.108277

[46] Wang, P.-W., Lin, H.-C., Yang, Y.-H. C., Hsu, C.Y., Chung, K.-S., Wu, H.-C., \& Yen, C.-F. (2017). Gender and Age Effects on the Trajectory of Depression in Opioid Users during Methadone Maintenance Treatment. Frontiers in Psychiatry, 8, 288-288. https://doi.org/10.3389/fpsyt.2017.00288

[47] Darke, S., Ross, J., Lynskey, M., \& Teesson, M. (2004). Attempted suicide among entrants to three treatment modalities for heroin dependence in the Australian Treatment Outcome Study (ATOS): prevalence and risk factors. Drug and Alcohol Dependence, 73(1), 1-10. https://doi.org/10.1016/j.drugalcdep.2003.08.008

[48] Darke, S., Williamson, A., Ross, J., \& Teesson, M. (2005). Attempted suicide among heroin users: 12month outcomes from the Australian Treatment Outcome Study (ATOS). Drug and Alcohol Dependence, 78(2), 177-186. https://doi.org/10.1016/j.drugalcdep.2004.10.009

[49] Friesen, E. L., \& Kurdyak, P. (2020). The impact of psychiatric comorbidity on treatment discontinuation among individuals receiving medications for opioid use disorder. Drug and Alcohol Dependence, 216, 108244-. https://doi.org/10.1016/j.drugalcdep.2020.108244 